\title{
Isla de patogenicidad de Vibrio parahaemolyticus en cepas chilenas clínicas y ambientales
}

\author{
H arold N úñeza, María Teresa Ulloaa, Fabiola Guerrab, \\ Carlos G O sorio.
}

Pathogenicity island region of clinical and environmental strains of Vibrio parahaemolyticus, isolated in Chile

Background: Most clinical isolates of Vibrio parahaemolyticus produce a major virulence factor known as the thermostable direct hemolysin (TDH). TDH is encoded by the tdh gene which is located in a genomic pathogenicity island (PAI). Most environmental isolates are described as tdh negative. Aim: To assess if environmental strains lack the full pathogenicity island or if only the tdh gene is deleted. Material and methods: Thirty eight clinical and 66 environmental strains of Vibrio parahaemolyticus were studied. PAI was characterized by polymerase chain reaction (PCR). The presence of tdhA and tdhS genes, was determined by Southern blot. Results: Fifty three environmental strains (80\%) lacked a full PAI when compared with clinical strains. In environmental strains, Southern blot and sequence analysis showed that a genetic region of 80 kilobase pairs including genes from VPA1310 to VPA1396 was missing. Conclusions: These results highlight the genetic dynamism of Vibrio parahaemolyticus pathogenecity island region and suggest that new pathogenic strains could appear by horizontal transfer of the island between toxigenic and non-toxigenic strains (Rev Méd Chile 2009; 137: 208-14).

(Key words: Genome, viral; Hemolysis, Vibrio; Vibrio parahaemolyticus)

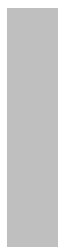

Recibido el 15 de julio, 2008. Aceptado el 13 de octubre, 2008.

Fuente de financiamiento: Proyecto de Reinserción de la Universidad de Chile (REIN 01/ 2004).

Programa de Microbiología y Micología, Instituto de Ciencias Biomédicas (ICBM), Facultad de Medicina, Universidad de Chile, Santiago, Chile.

aTecnólogo Médico

${ }^{\mathrm{b}}$ Médico Veterinario

Correspondencia a: Dr. Carlos Osorio. Programa de Microbiología y Micología, Facultad de Medicina, Universidad de Chile, Av. Independencia 1027, Santiago, Chile. Teléfono: 56-2-978 6902; Fax: 56-2-735 5855.

E mail: gonosorio@med.uchile.cl 
$\mathrm{V}$ ibrio parahaemolyticus es una bacteria Gram negativa, perteneciente al phylum Gammaproteobacteria, halofílica, mótil y habita normalmente en ambientes marinos. Las cepas toxigénicas de este vibrión causan un cuadro de gastroenteritis aguda autolimitado, asociado frecuentemente al consumo de mariscos crudos. Las cepas toxigénicas se caracterizan por codificar en su genoma para dos importantes factores de virulencia de este vibrión, la denominada hemolisina directa termoestable (TDH) y la hemolisina directa termoestable relacionada (TRH). Estas dos hemolisinas se pueden encontrar simultáneamente o por separado en cepas toxigénicas de $\mathrm{V}$ parahaemolyticus, correlacionándose ambas fuertemente con la capacidad patogénica de las cepas portadoras $^{1-3}$. En contraste, la mayoría de las cepas ambientales aisladas desde moluscos bivalvos o del ambiente marino corresponden a cepas no toxigénicas que carecen de ambas hemolisinas (TDH y TRH $)^{4}$. La caracterización de cepas aisladas desde casos clínicos y mariscos en Chile durante el período 1998-2007 ha mostrado este mismo patrón ${ }^{5-7}$. Recientemente, el genoma de la cepa pandémica toxigénica de $\mathrm{V}$ parahaemolyticus RIMD2210633 fue secuenciado, demostrándose que ella carece del gen codificante para TRH, pero que porta una isla de patogenicidad (IPA) en su cromosoma menor que contiene dos genes codificantes para TDH (tdhA y tdhS $)^{8}$. La ausencia de TDH en las cepas ambientales se podría explicar por tanto, por ausencia de la IPA completa (incluyendo ambos genes tdh) o alternativamente por una deleción delimitada únicamente a estos dos genes. Apoyando esta última alternativa, se ha reportado que algunas IPA de otras especies bacterianas presentan una alta inestabilidad genética, sufriendo frecuentes deleciones de algunos segmentos 9 . Para contrastar estas alternativas, fueron caracterizadas genéticamente las IPA de 38 cepas clínicas y 66 cepas ambientales de V parahaemolyticus aisladas desde pacientes y mariscos en Chile, respectivamente, durante el período 1998-2007.

\section{Material y MÉTODO}

Cepas bacterianas y condiciones de cultivo. Las cepas clínicas y ambientales de $\mathrm{V}$ parahaemolyticus usadas en este estudio fueron provistas amablemente por el Dr. Romilio Espejo, del Instituto de Nutrición y Tecnología de Alimentos (INTA) de la Universidad de Chile, por el Dr. Juan Carlos Hormazábal, del Instituto de Salud Pública de Chile (ISP) y por el SEREMI de Salud de la Región Metropolitana. Las cepas clínicas corresponden a cepas aisladas en el periodo 1998-2005 en diferentes localidades: Puerto Montt, Iquique, Arica, Talcahuano, Coquimbo y Concepción. Las cepas ambientales corresponden a cepas aisladas desde mariscos entre los años 2003 y 2007 desde regiones y localidades como: Llanchipal, Araucanía, Copiapó, Taltal, Región Metropolitana, Chullín y Pelluco.

Las cepas clínicas fueron designadas C1-C38 y las ambientales fueron denominadas A1-A66. La cepa de referencia RIMD2210633 fue obtenida directamente del Research Institute for Microbial Diseases (RIMD), Osaka, Japón. Todas las cepas fueron crecidas en caldo Luria-Bertani (LB) suplementado con $\mathrm{NaCl} 2 \%$, agar LB suplementado con $2 \%$ de $\mathrm{NaCl}$ y agar tiosulfato-citrato-sales biliaressacarosa (TCBS) por aproximadamente $12 \mathrm{~h}$ a $37^{\circ} \mathrm{C}$. Para observar su capacidad hemolítica, las cepas fueron crecidas en agar Wagatsuma modificado, preparado de la siguiente forma: $3 \mathrm{~g}$ de extracto de levadura, $10 \mathrm{~g}$ de peptona, $70 \mathrm{~g}$ de $\mathrm{NaCl}, 5 \mathrm{~g}$ de $\mathrm{K}_{2} \mathrm{HPO}_{4}, 10 \mathrm{~g}$ de manitol, $10 \mathrm{~g}$ de agar, 11 de agua destilada y $50 \mathrm{ml}$ de sangre humana desfibrinilada. Todas las cepas fueron observadas en un microscopio de contraste de fases $\left(\right.$ Zeiss ${ }^{\circledR}$ ) con aumento 100X y fueron sometidas posteriormente a la prueba de la oxidasa $\left(\right.$ Oxoid $^{\circledR}$ ) y a una batería API-20E para enterobacterias (BioMerieu ${ }^{\circledR}$ ).

Ensayos de PCR y secuenciación. Para caracterizar la IPA de V parahaemolyticus se realizaron primeramente ensayos de reacción en cadena de la polimerasa (PCR) utilizando un conjunto de partidores que se muestra en la Tabla 1 (ver detalles en Figura 1). Todos los partidores se confeccionaron en base a la secuencia publicada del genoma de la cepa pandémica RIMD22106333. Cada reacción se realizó en un volumen total de $20 \mu \mathrm{l}$, conteniendo 1 $\mathrm{mM}$ de mezcla de dNTPs, 3 pmol de cada partidor, 1,5 $\mathrm{mM}$ de $\mathrm{MgCl}_{2}, 1 \mathrm{x}$ de buffer de reacción (10 mM Tris-HCl, $50 \mathrm{mM} \mathrm{KCl}$ ), 2,5 U de Taq ADN polimerasa $\left(\right.$ Invitrogen $\left.{ }^{\circledR}\right)$, y $1 \mu$ l de ADN genómico de cada 
Tabla 1. Partidores usados en la caracterización de la isla de patogenicidad de cepas clínicas y ambientales de V parahaemolyticus ${ }^{\mathrm{a}}$

\begin{tabular}{|llcc|}
\hline Partidor & Secuencia 5'-3' & Producto (bp) & Referencia \\
\hline tdhAf & GTACCGATATTTTGCAAA & 381 & $(15)$ \\
tdhAr & ATGTTGAAGCTGTACTTGA & & $(15)$ \\
tdhA1f & TTTGAGCTTCCATCTGTC & 281 & Estudio actual \\
tdhA1r & ACAGCAGAATGACCGTG & & Estudio actual \\
vpa1355f & CCA GCA GTG TGA TGA TTG & 1727 & Estudio actual \\
vpa1355r & CAT TTT CAT TGA CCA CGA C & 750 & Estudio actual \\
vpa1380f & ATG GTA TCA ATG AAA CTA TC & & Estudio actual \\
vpa1380r & TTC TCA AAT GGG TAA GTG & 3177 & Estudio actual \\
vpa1304f & CAC AAA TTG ACT TGA TAA CTG & & Estudio actual \\
vpa1308r & CGT CAT AAC TCA GAG CGA G & & Estudio actual \\
vpa1397f & ATT GGT GAA GAG ATG GAA G & ca. 1700 & Estudio actual \\
vpa1400r & ACT AAG GAG AGA TTA CAA TG & & Estudio actual \\
vpa1308f & C TCG CTC TGA GTT ATG ACG & ca. 1000 & Estudio actual \\
vpa1397r & CTT CCA TCT CTT CAC CAA T & & Estudio actual \\
FABI5 & CAA GTG ATG CCT TTA CAT GA & & \\
vpa1397r & CTT CCA TCT CTT CAC CAA T & & \\
\hline
\end{tabular}

aTodos los partidores usados en este trabajo se diseñaron en base al genoma de la cepa pandémica V. parahaemolyticus RIMD2210633 obtenido desde el sitio TIGR.

Figura 1. Esquema de la isla de patogenicidad de la cepa pandémica RIMD2210633 de V parahaemolyticus (1A) y de la misma región de cepas ambientales no toxigénicas de V. parahaemolyticus (1B). Los partidores están definidos en la Tabla 1. Sólo cuatro de los 89 genes putativos de la IPA se ilustran en la Figura 1A.

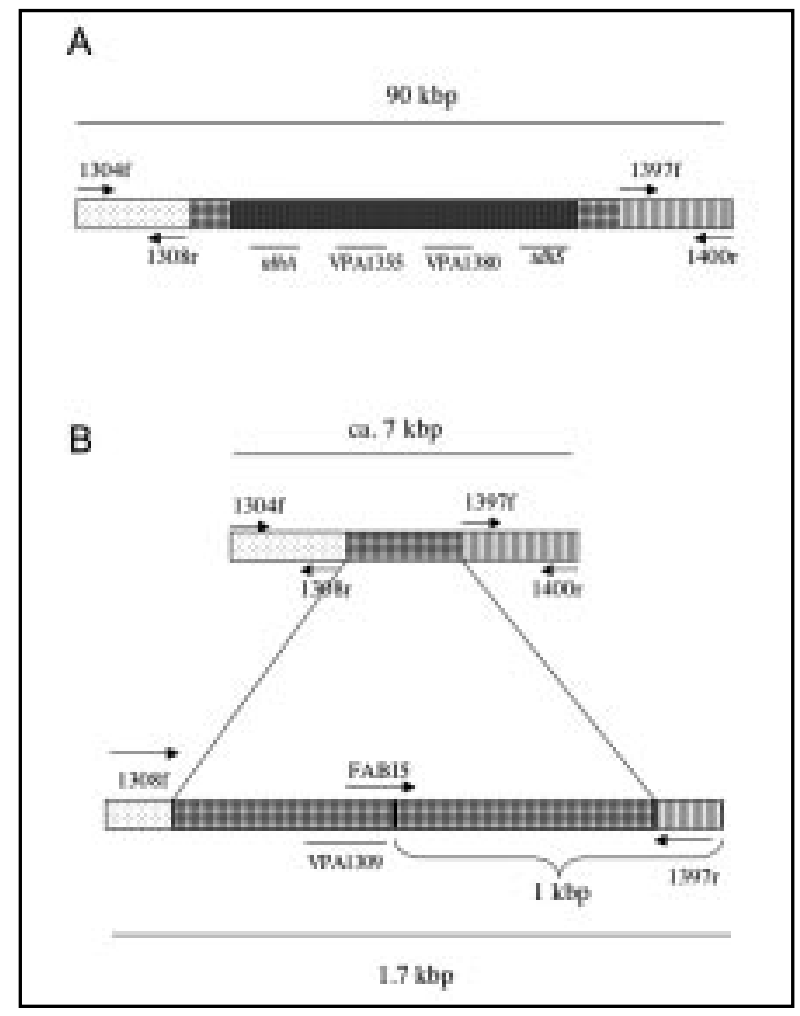


cepa como templado. El ADN genómico se obtuvo usando el kit de purificación de ADN genómico DNeasy Tissue (Qiagen ${ }^{\circledR}$ ). Cada amplificación se realizó de acuerdo al siguiente protocolo de hot start»: primero la mezcla se calentó a $96^{\circ} \mathrm{C}$ por 3 min y luego se agregó la enzima Taq polimerasa (Invitrogen ${ }^{\circledR}$ ). Posteriormente se aplicaron 30 ciclos de amplificación, consistiendo cada uno de ellos en: $30 \mathrm{~s}$ a $95^{\circ} \mathrm{C}, 30 \mathrm{~s}$ a $55-60^{\circ} \mathrm{C}$ y $1 \mathrm{~min}$ a $72^{\circ} \mathrm{C}$ (rango: 1-7 min, dependiendo del tamaño del producto esperado). Los amplicones fueron separados por electroforesis en geles de agarosa a 1,5\% y visualizados con bromuro de etidio. Los amplicones que fueron sometidos a secuenciación fueron purificados usando el kit QIAquick Gel Extraction $\left(\right.$ Qiagen $\left.^{\circledR}\right)$.

Ensayos de Southern blot. Los ensayos de Southern blot fueron realizados de acuerdo a Sambrock et al, $1989^{10}$. El ADN genómico fue purificado usando el kit de purificación DNeasy Tissue $\left(\right.$ Qiagen ${ }^{\circledR}$ ) y luego digerido con la enzima de restricción EcoRI.
El tamaño de los dos fragmentos de hibridación esperados en estas condiciones para las cepas toxigénicas es de 1,1 y 4,3 kpb. La sonda de ADN utilizada fue dirigida para la región común de 381 pb de los genes tdhA y tdhSy fue sintetizada con el par de partidores tdhAf-tdhAr (ver Tabla 1). Luego la sonda fue marcada con el isótopo ${ }^{32} \mathrm{P}$ utilizando el kit Megaprime DNA Labeling System (Amersham Bioscience ${ }^{\circledR}$ ). Las placas fueron expuestas por $2 \mathrm{~h} \mathrm{y}$ luego leídas con un equipo PhosphoImager (Bio$\left.\operatorname{Rad}^{\circledR}\right)$.

\section{Resultados}

Del total de 38 cepas clínicas estudiadas todas resultaron ser toxigénicas en base a la presencia de los genes tdhA y tdhS y a la capacidad hemolítica evidenciada en agar Wagatsuma (fenómeno de Kanagawa). Por otra parte, las 66 cepas ambientales estudiadas fueron del tipo no toxigénicas y no hemolíticas (ver Tabla 2).

\section{Tabla 2. C aracterísticas genéticas y hemolíticas de cepas chilenas clínicas y ambientales de Vibrio parahaemolyticus aisladas en Chile en el período 1998-2007*}

\begin{tabular}{|lcc|}
\hline Propiedad o característica & $\begin{array}{c}\text { Número de cepas con la propiedad, aisladas de* } \\
\text { Mariscos (A1-A66) }\end{array}$ & $\begin{array}{c}\text { Clínicas (C1-C38) } \\
\text { Detección de tdh por }\end{array}$ \\
$\begin{array}{l}\text { PCR }^{\dagger} \\
\text { Sonda }\end{array}$ & $0 / 66$ & $37 / 38$ \\
Fenómeno de Kanagawa positivo & $0 / 18$ & $10 / 10$ \\
Amplificación por PCR de extremos & & \\
1304-1308 & $0 / 18$ & $38 / 38$ \\
1397-1400 & & $38 / 38$ \\
Amplificación por PCR de segmento & $59 / 66$ & \\
FABI5-VPA1397प & $47 / 50$ & $0 / 38$ \\
\hline
\end{tabular}

*Todos los resultados se expresan como № de cepas positivas/№ total de cepas estudiadas

${ }^{\dagger}$ Detección por PCR del gen tdhA usando el par de partidores tdhAf/tdhAr

‡Southern blot usando una sonda radioactiva de $300 \mathrm{pb}$ para los genes tdhA and tdhS

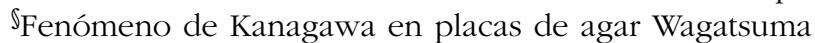

†Detección por PCR de extremos de isla de patogenicidad usando los pares de partidores VPA1304f-

VPA1308r y VPA1397f-VPA1400r

"Detección por PCR de segmento de $1 \mathrm{kbp}$ amplificado con par de partidores FABI5-vpa1397r 
Las reacciones de PCR para las cepas clínicas fueron positivas para los marcadores tdhA, segmento VPA1304f-VPA1308r (extremo 5' de la IPA) y segmento VPA1397f-VPA1400r (extremo 3' de la IPA) y marcadores tdhA1, VPA1355, VPA1380 (datos no mostrados), consistente con su estrecha relación genética con la cepa pandémica RIMD2210633 ${ }^{11}$. En concordancia con los resultados anteriores, el ensayo de Southern-blot con la sonda radioactiva de ADN para los genes tdhA y tdhS determinó la presencia de dos bandas de un tamaño esperado de 1,1 y 4,3 kpb (datos no mostrados). En claro contraste, la mayoría de las cepas ambientales (53/66, aproximadamente 80\%) fueron negativas para los marcadores internos de la IPA (e.g., tdh, VPA1355, VPA1380) y no demostraron bandas de hibridación en los ensayos de Southern-blot. Sin embargo, es destacable que estas cepas fueron positivas por PCR para los extremos 5' y 3' de la IPA. Desde estas cepas se amplificó además un producto de $1 \mathrm{kpb}$ con el par de partidores FABI5-VPA1397r (Tabla 2). Es importante destacar aquí que el partidor FABI5 se encuentra dentro del marco de lectura abierto VPA1309 (Figura 1A y 1B). Basándose en estos resultados y en información previa sobre los perfiles de campo pulsado de las cepas ambientales estudiadas en este trabajo (Hormazábal, J.C., resultados no publicados), se seleccionaron 3 cepas ambientales de perfiles claramente distantes para secuenciar el amplicón de $1 \mathrm{kpb}$ arriba mencionado. La zona central del alineamiento de estas secuencias se muestra en la Figura 2. Este resultado confirma que las cepas secuenciadas carecen completamente de la región de $80 \mathrm{kpb}$ entre los genes VPA1309 y VPA1397.

\section{DisCuSIÓN}

Los resultados obtenidos en este trabajo demuestran que gran parte de las cepas ambientales carecen de la región central de la IPA, conservando sus extremos $5^{\prime}$ y $3^{\prime}$ intactos (segmentos VPA1304-VPA1308 y VPA1397-VPA1400, respectivamente). Los resultados de PCR se complementan perfectamente con los resultados de la secuenciación, indicando específicamente que el segmento genético que se extiende desde el gen VPA1309 al gen VPA1396, abarcando una extensión de casi $80 \mathrm{kpb}$ y aproximadamente 86 genes putativos (segmento VPA1310-VPA1396), no se encuentra presente en la mayoría de las cepas ambientales. Por otra parte, estudios previos de PFGE (Hormazábal, J.C., resultados no publicados) indican que las cepas ambientales estudiadas presentan una amplia diversidad genética. Esto indica que la ausencia de la IPA en las cepas ambientales es un fenómeno extendido y que no se debe a un sesgo del muestreo. Apoya esta interpretación, el hecho que el conjunto de cepas ambientales presenta una amplia distribución res-

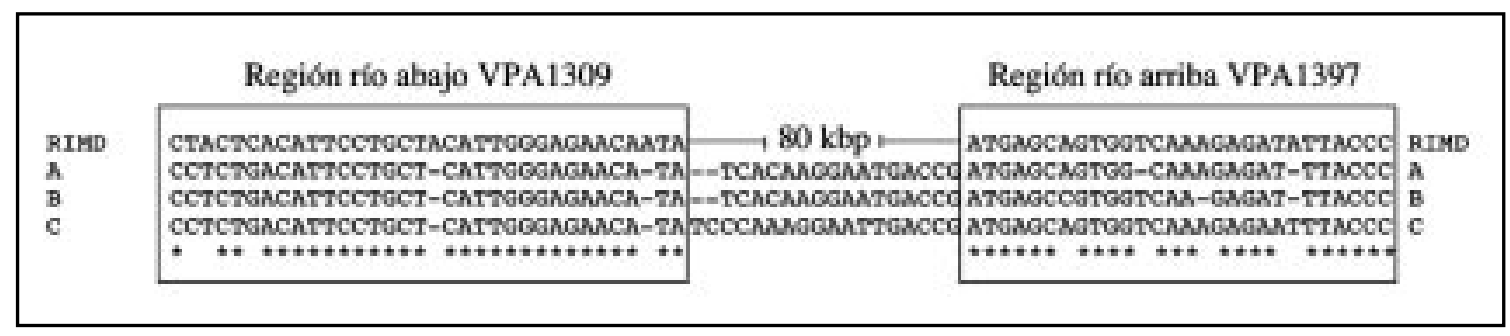

Figura 2. Alineamiento de secuencias obtenidas con el par de partidores FABI5-VPA1397r desde cepas ambientales no toxigénicas de V parahaemolyticus. Se amplificó por PCR con los partidores FABI5 y 1397r y luego se secuenció el fragmento obtenido (el alineamiento se realizó con el programa CLUSTALW). A, B y C: secuencias obtenidas para las cepas ambientales A3, A5 y A7, respectivamente; RIMD: secuencia de la cepa pandémica RIMD2210633 de V parahaemolyticus. Los rectángulos indican las regiones río abajo y río arriba de los genes VPA1309 y VPA1397, respectivamente. Los asteriscos indican residuos nucleotídicos idénticos a la cepa RIMD2210633. Se debe destacar que sólo se muestra la región central del alineamiento. 
pecto al lugar geográfico (Ej: Antofagasta, Concepción y Puerto Montt) y la fecha de su recolección (1998-2007). Por otra parte, nuestros resultados no permiten inferir la integridad de la IPA en las cepas clínicas estudiadas, debido a que en ellas lo único que es posible observar es la presencia de los genes tdhA, tdhS, VPA1355 y VPA1380, y la ausencia del amplicón esperado de $1 \mathrm{kpb}$ generado con el par FABI5-VPA1397r. Es posible que existan pequeñas o medianas deleciones en la IPA de estas cepas y que no sean detectadas mediante esta estrategia. Destaca que los extremos de la IPA se encuentren presentes tanto en cepas clínicas como en cepas ambientales. Ello plantea la posibilidad de que los extremos de la IPA estén mal definidos. Lo que en este trabajo denominamos extremos 5 y $3{ }^{\prime}$ de la IPA pudieran corresponder más bien a las regiones $5^{\prime}$ y 3 ' adyacentes a la IPA propiamente tal. Esta indeterminación se debe, al menos en parte, a que la IPA de V parahaemolyticus no está inserta en un gen de tARN y no presenta secuencias repetidas directas claramente identificables en sus márgenes $^{8}$. Los resultados de este trabajo favorecen la interpretación de que la IPA está ausente en cepas ambientales y que sus extremos deben ser redefi-

\section{REFERENCIAS}

1. Nishibuchi M, Kaper JB. Nucleotide sequence of the thermostable direct hemolysin gene of Vibrio parahaemolyticus. J Bacterial 1985; 162: 558-64.

2. Nishibuchi M, KaPER JB. Thermostable direct hemolysin gene of Vibrio parahaemolyticus: a virulence gene acquired by a marine bacterium. Infect Immun 1995; 63: 2093-9.

3. Nishibuchi M, Taniguchi T, Misawa T, Khaeomanee-Iam V, Honda T, Miwatani T. Cloning and nucleotide sequence of the gene (trh) encoding the hemolysin related to the thermostable direct hemolysin of Vibrio parahaemolyticus. Infect Immun 1989; 57: 2691-7.

4. Nishibuchi M, Ishibashi M, Takeda Y, Kaper JB. Detection of the thermostable direct hemolysin gene and related DNA sequences in Vibrio parahaemolyticus and other vibrio species by the DNA colony hybridization test. Infect Immun 1985; 49: 481-6.

5. Fuenzalida l, Armijo L, Zabala B, Hernández C, Rioseco ML, Riquelme C et al. Vibrio parahaemolyticus strains nidos. Estos complementan perfectamente estudios previos que mostraban que la mayoría de las cepas ambientales de $\mathrm{V}$ parahaemolyticus son del tipo no patogénico ${ }^{4-6}$, estableciendo una base genética para dicha observación que no había sido descrita previamente en la literatura. Durante la preparación de este manuscrito, el grupo de Honda et $a^{12,13}$ reportó que la mayoría de las cepas ambientales estudiadas por ellos carece de la región central de la IPA (conservando sus extremos), lo que concuerda plenamente con nuestros resultados y establece la necesidad de redefinir los bordes o límites de esta estructura. Por otra parte, los resultados obtenidos nos llevan a plantear la posibilidad que la IPA de V parahaemolyticus pueda transferirse horizontalmente entre cepas toxigénicas y ambientales no toxigénicas, pudiendo así surgir nuevas variantes con diferente potencial patogénico ${ }^{14}$.

\section{Agradecimientos}

A los Drs. Romilio Espejo, del INTA (Universidad de Chile) y Juan Carlos Hormazábal, del Instituto de Salud Pública por proveernos de gran parte de las cepas utilizadas en este trabajo. isolated during investigation of the summer 2006 seafood related diarrhea outbreaks in two regions of Chile. Int J Food Microbiol 2007; 117: 270-5.

6. Fuenzalida L, Hernández C, Toro J, Rioseco ML, Romero J, Espejo RT. Vibrio parahaemolyticus in shellfish and clinical samples during two large epidemics of diarrhoea in southern Chile. Environ Microbiol 2006; 8: 675-83.

7. Nair GB, Hormazabal JC. The Vibrio parahaemolyticus pandemic. Rev Chilena Infectol 2005; 22: 125-30.

8. Makino K, Oshima K, Kurokawa K, Yokoyama K, Uda T, TAgOMORI K ET AL. Genome sequence of Vibrio parahaemolyticus: a pathogenic mechanism distinct from that of V. cholerae. Lancet 2003; 361: 743-9.

9. Blue $G$, Оtt M, Lischewski A, Ritter A, Imrich $H$, Tschape $H$ ET aL. Excision of large DNA regions termed pathogenicity islands from tRNA-specific loci in the chromosome of an Escherichia coli wild-type pathogen. Infect Immun 1994; 62: 606-14.

10. Sambrook J, Fritsch EF, Maniatis T. Molecular Cloning: A Laboratory Manual, $2^{\text {th }}$ Ed, Cold Spring Harbor 
Laboratory Press, 1989.

11. Gonzalez-Escalona N, Cachicas V, Acevedo C, Rioseco ML, Vergara Ja, Cabello F, Romero J et al. Vibrio parahaemolyticus diarrhea, Chile, 1998 and 2004. Emerg Infect Dis 2005; 11: 129-31.

12. Izutsu K, Kurokawa K, Tashiro K, Buhara S, Hayashi T, Honda T ET AL. Comparative genomic analysis using microarray demonstrates a strong correlation between the presence of the 80-kilobase pathogenicity island and pathogenicity in Kanagawa phenomenon-positive Vibrio parahaemolyticus strains. Infect Immun 2008; 76: 1016-23.
13. Sugryama T, Iida $T$, IzuTsu $K$, Park KS, Honda $T$. Precise region and the character of the pathogenicity island in clinical Vibrio parahaemolyticus strains. J Bacteriol 2008; 190: 1835-7.

14. Lesic B, Carniel E. Horizontal Transfer of the HighPathogenicity Island of Yersinia pseudotuberculosis. J Bacteriol 2005; 187: 3352-8.

15. Suthienkul O, Ishibashi M, Iida T, Nettip N, Supavej $\mathrm{S}$, EAMPOKALAP B ET AL. Urease production correlates with possession of the trh gene in Vibrio parahaemolyticus strains isolated in Thailand. J Infect Dis 1995; 172: 1405-8. 